\title{
On Lerch's class number formulae for binary quadratic forms
}

\author{
by L. J. MordeLL
}

Let $f(-d)$ be the number of classes of positive quadratic forms $a x^{2}+2 b x y+c y^{2}$ with negative determinant $-d=b^{2}-a c$, where $a$ and $c$ are not both even and $(a$, $b, c)=1$. It is well known that if $\dot{d} \neq 1$,

$$
f(-d)=\frac{2 \sqrt{d}}{\pi} \sum_{\substack{n=1 \\ n \text { odd }}}^{\infty} \frac{1}{n}\left(\frac{-d}{n}\right),
$$

and that when $d$ is a prime $p \equiv 3(\bmod 4)$, this can be written as

$$
f(-p)=\sum_{n=1}^{i(p-1)}\left(\frac{n}{p}\right) .
$$

Here $(n / p)$ is the Legendre-Jacobi symbol. There are other formulae expressing partial sums of quadratic residues in terms of $f(-p), f(-2 p), f(-3 p)$. Such results were known to Gauss.

Many more interesting formulae of this kind have been given by Lerch [1] in his Paris Academy prize memoir for 1900 . He, however, deals with Kronecker forms $a x^{2}+b x y+c y^{2}$ whose discriminant $b^{2}-4 a c$, when positive is written as $D$, and when negative as $-\Delta$. The corresponding class number $h(-\Delta)$ of forms with $(a, b, c)=1$ can be written, when $-\Delta$ is a fundamental discriminant and $\Delta>4$, as

$$
h(-\Delta)=-\frac{1}{\Delta} \sum_{n=1}^{\Delta}\left(\frac{-\Delta}{n}\right) n .
$$

The symbol $(-\Delta / n)$ is now and hereafter the Kronecker symbol. We note that $(-\Delta / m)=(-\Delta / n)$ sgn $m n$ if $m \equiv n(\bmod \Delta)$ and $-\Delta$ is a negative discriminant, and that $(D / m)=(D / n)$ if $m \equiv \pm n(\bmod D)$ and $D$ is a positive discriminant.

He then deduces many formulae of the type (2) from his

Theorem I. Let $D>0$, and $-\Delta<0$ be two fundamental discriminants with $(D, \Delta)$ $=1$. Then

$$
h(-\Delta D)=2 \sum_{a=1}^{+\Delta}\left(\frac{-\Delta}{a}\right) \sum_{n=1}^{a D / \Delta}\left(\frac{D}{n}\right)
$$


L. J. MORDELL, On Lerch's class number formula for binary quadratic forms

$$
-h(-\Delta D)=2 \sum_{a=1}^{1} D\left(\frac{D}{a}\right) \sum_{n=1}^{a \Delta / D}\left(\frac{-\Delta}{n}\right) .
$$

A sum such as $\sum_{n=1}^{N}$ means $1 \leqslant n \leqslant[N]$ where $[N]$ is the integer part of $N$.

Theorem II. Let $D_{1}, D_{2}, \ldots, D_{r}$ denote fundamental discriminants relatively prime in pairs with absolute values $\Delta_{1}, \ldots, \Delta_{r}$ and with $2 s+1$ of the $D$ negative. Then

$$
h\left(D_{1} D_{2} \ldots D_{r}\right)=(-1)^{s} \sum\left(\frac{D_{1}}{n_{1}}\right)\left(\frac{D_{2}}{u_{2}}\right) \ldots\left(\frac{D_{r}}{n_{r}}\right)\left[\frac{n_{1}}{\Delta_{1}}+\frac{n_{2}}{\Delta_{2}}+-+\frac{n_{r}}{\Delta_{r}}\right],
$$

where the square bracket denotes the integer part and the summation is taken over

$$
0<n_{1}<\Delta_{1}, \ldots, 0<n_{r}<\Delta_{r} .
$$

In the enunciation of Theorem I, Lerch does not state emplicitly that $(D, \Delta)=1$, but this is tacitly assumed on page 405 [2] in his formula for $h(-\Delta D)$ as a Fourier series. Similarly in Theorem II, he omits the conditions $\left(D_{1}, D_{2}\right)=1$, etc., but without these, his argument on page 374 [3], stating that a certain sum is zero, is not valid.

Lerch's proof of Theorem $I$ is rather complicated involving various transformations of infinite series. His proof of Theorem II is also much longer than seems to be necessary. It may be worth while pointing out that $(4),(5)$ are particular cases of (6), and that (6) is a simple consequence of (3). In (3), write $-\Delta=D_{1} D_{2} \ldots D_{r}$ where the $D$ are as in Theorem II. In (3), $n$ runs through a complete set of positive residues $\bmod \Delta$. A complete set of positive residues $\bmod \Delta$ is given by

$$
R=\Delta\left(\frac{n_{1}}{\Delta_{1}}+\frac{n_{2}}{\Delta_{2}}+\ldots+\frac{n_{r}}{\Delta_{r}}\right), \quad 0 \leqslant n_{1}<\Delta_{1}, \ldots, 0 \leqslant n_{r}<\Delta_{r} .
$$

These numbers, however, are not all less than $\Delta$ and so we cannot replace $n$ by $R$. We can, however, put

$$
n=R-\varepsilon_{t} \Delta,
$$

where $\varepsilon_{t}=t,(t=0,1, \ldots, r-1)$ when $t \leqslant R / \Delta<t+1$, i.e., $\varepsilon_{t}=[R / \Delta]$.

Now

$$
\left(\frac{-\Delta}{n}\right)=\left(\frac{D_{1}}{n}\right) \ldots\left(\frac{D_{r}}{n}\right)=\prod\left(\frac{D_{1}}{n}\right)
$$

say. Also when $A \Delta_{1}+B>0, B>0$,

$$
\left(\frac{D_{1}}{A \Delta_{1}+B}\right)=\left(\frac{D_{1}}{B}\right)
$$

Hence

$$
\left(\frac{-\Delta}{n}\right)=\prod\left(\frac{D_{1}}{\Delta_{2} \ldots \Delta_{r}}\right)\left(\frac{D_{1}}{n_{1}}\right)=\prod\left(\frac{D_{1}}{\Delta_{2}}\right)\left(\frac{D_{2}}{\Delta_{1}}\right)\left(\frac{D_{1}}{n_{1}}\right)
$$

say. By the law of quadratic reciprocity, this becomes 


$$
\begin{aligned}
\left(\frac{-\Delta}{n}\right) & =\prod(-1)^{\frac{1-\operatorname{sgn} D_{1}}{2}} \cdot \frac{1-\operatorname{sgn} D_{2}}{2} \Pi\left(\frac{D_{1}}{n_{1}}\right) \\
& =(-1)^{\frac{(2 s+1) 2 s}{2}} \prod\left(\frac{D_{1}}{n_{1}}\right) \\
& =(-1)^{s} \prod\left(\frac{D_{1}}{n_{1}}\right) .
\end{aligned}
$$

Hence $\quad-\Delta h(-\Delta)=(-1)^{s} \sum\left(\frac{D_{1}}{n_{1}}\right) \ldots\left(\frac{D_{r}}{n_{r}}\right)\left(\Delta\left(\frac{n_{1}}{\Delta_{1}}+\ldots+\frac{n_{2}}{\Delta_{2}}\right)-\varepsilon_{t} \Delta\right)$.

Since $\sum\left(D_{1} / n_{1}\right)=0$, etc., the first part of the sum is zero and we have $(6)$, namely,

$$
h(-\Delta)=\sum\left(\frac{D_{1}}{n_{1}}\right) \ldots\left(\frac{D_{r}}{n_{r}}\right)\left[\frac{n_{1}}{\Delta_{1}}+\ldots+\frac{n_{r}}{\Delta_{r}}\right] .
$$

We now deduce (4), (5). Take $r=2,-\Delta=D_{1} D_{2}$ and so $s=0$.

Then

$$
h\left(D_{1} D_{2}\right)=\sum\left(\frac{D_{1}}{n_{1}}\right)\left(\frac{D_{2}}{n_{2}}\right)\left[\frac{n_{1}}{\Delta_{1}}+\frac{n_{2}}{\Delta_{2}}\right] .
$$

Replace $n_{1}$ by $\Delta_{1}-n_{1}$. Then (7) becomes

$$
\begin{aligned}
h\left(D_{1} D_{2}\right) & =\sum\left(\frac{D_{1}}{\Delta-n_{1}}\right)\left(\frac{D_{2}}{n_{2}}\right)\left(\frac{n_{2}}{\Delta_{2}}-\frac{n_{1}}{\Delta_{1}}+1\right) \\
& =\operatorname{sgn} D_{1} \sum\left(\frac{D_{1}}{n_{1}}\right)\left(\frac{D_{2}}{n_{2}}\right)\left[\frac{n_{2}}{\Delta_{2}}-\frac{n_{1}}{\Delta_{1}}+1\right] .
\end{aligned}
$$

The sum is zero unless $n_{1} / \Delta_{1}<n_{2} / \Delta_{2}$, and then we have

$$
h\left(D_{1} D_{2}\right)=\operatorname{sgn} D_{1} \sum_{n_{2}=1}^{\Delta_{2}}\left(\frac{D_{2}}{n_{2}}\right) \sum_{n_{1}=1}^{\Delta_{1} n_{2} / \Delta_{2}}\left(\frac{D_{1}}{n_{1}}\right) .
$$

For $D_{1}=D, D_{2}=-\Delta$, this becomes

$$
h(-D \Delta)=\sum_{n_{2}=1}^{\Delta}\left(\frac{-\Delta}{n_{2}}\right) \sum_{n_{1}=1}^{n_{2} D / \Delta}\left(\frac{D}{n_{1}}\right) .
$$

For $D_{1}=-\Delta, D_{2}=D$, this becomes

$$
h(-D \Delta)=-\sum_{n_{2}=1}^{D}\left(\frac{D}{n_{2}}\right) \sum_{n_{1}=1}^{n_{2} \Delta / D}\left(\frac{-\Delta}{n_{1}}\right) .
$$

We show now that the $n_{2}$ summation in (8) and (9) need only be taken to $\frac{1}{2} \Delta$, $\frac{1}{2} D$ respectively if we insert a factor 2 on the right-hand sides of (8) and (9). We have then the results (4) and (5). It suffices to show that the sum (8) remains unaltered if we replace $n_{2}$ by $\Delta-n_{2}$. For then with $(\Delta / 0)=0$, etc., 
L. J. MORDELL, On Lerch's class number formula for binary quadratic forms

$$
\sum_{n_{1}=1}^{\Delta}=\sum_{n_{2}=0}^{1 \Delta}
$$

Also

$$
\left(\frac{-\Delta}{\Delta-n_{2}}\right)=\left(\frac{-\Delta}{n_{2}}\right) \text {, }
$$

and the $n_{1}$ sum in (8) becomes

$$
\sum_{n_{1}=1}^{D-n_{2} D / \Delta}\left(\frac{D}{n_{1}}\right) .
$$

Replace now $n_{1}$ by $D-n_{1}$. Then

$$
\left(\frac{D}{D-n_{1}}\right)=\left(\frac{D}{n_{1}}\right)
$$

and the sum becomes

since

$$
\begin{gathered}
\sum_{n_{1}=n_{2} D / \Delta}^{D}\left(\frac{D}{n_{1}}\right)=-\sum_{n_{1}=1}^{n_{2} D / \Delta}\left(\frac{D}{n_{1}}\right) \\
\sum_{n_{1}=1}^{D}\left(\frac{D}{n_{1}}\right)=0 .
\end{gathered}
$$

This finishes the proof.

This research was supported in part by the National Science Foundation. Washington, D.C.

University of Arizona, Tucson, Arizona U.S.A. and St. Johns College, Cambridge, England.

\section{REFERENCES}

1. Lerch, Essais sur le calcul du nombre des classes de forms quadratiques binaires aux coefficients entiers. Acta Mathematica 29 (1905) 333-424 (in particular page 407) and 30 (1906) 203-294.

2. Ibid., Vol. 29.

3. Ibid., Vol. 29. 